Eurasian Journal of Physics and Functional Materials

2021, 5(4), 198-210

\title{
Investigation and diagnostics of plasma flows in a pulsed plasma accelerator for experimental modelling of processes in tokamaks
}

\author{
M.K. Dosbolayev, A.B. Tazhen*, T.S. Ramazanov \\ Al-Farabi Kazakh National University, Almaty, Kazakhstan \\ E-mail: tazhen.aigerim.b@gmail.com
}

DOI: $10.32523 /$ ejpfm.2021050404

Received: 20.10.2021 - after revision

\begin{abstract}
This paper presents the experimental results on electron, ion temperatures and densities in a pulsed plasma accelerator. The values of electron densities and temperatures were computed using the methods of relative intensities of $H_{\alpha}$ and $H_{\beta}$ lines, $H_{\beta}$ Stark broadening, and the technique is based on Faraday cup beam current measurements. In this work, a linear optical spectrometer S-100 was used to acquire the emission spectra of hydrogen and air plasmas. In this spectrum, there are some lines due to $\mathrm{Fe}, \mathrm{Cu}$, $\mathrm{N}_{2}, \mathrm{O}_{2}$, and $\mathrm{H}_{2}$. The series of visible lines in the hydrogen atom spectrum are named the Balmer series. The spectral emissions of iron and copper occur throughout the gas breakdown and ignition of an arc discharge, during the erosion and sputtering of materials. The vacuum chamber and coaxial electrodes were made. The electron temperatures and densities in a pulsed plasma accelerator, measured via relative intensities of spectral lines and Stark broadening, at a charging voltage of a capacitor bank of $3 \mathrm{kV}$ and a working gas pressure in a vacuum chamber of $40 \mathrm{mTorr}$, were $2.6 \mathrm{eV}$ and $1.66 \cdot 10^{16} \mathrm{~cm}^{-3}$ for hydrogen plasma. These results were compared with the Faraday cup beam current measurements. However, no match was found. Considering and analyzing this distinction, we concluded that the spectral method of plasma diagnostics provides more accurate results than electrical measurement. The theory of probe measurements can give approximate results in a moving plasma.
\end{abstract}

Keywords: pulsed plasma accelerator, plasma spectroscopy, plasma diagnostics, Stark broadening, Balmer series lines, spectral lines, Faraday cup

\section{Introduction}

It is costly to conduct serial scientific experiments in controlled thermonuclear fusion facilities. There are insufficient computational resources to simulate, for 
example, the interaction of the energetic plasma with the candidate materials. In this regard, modelling experiments are carried out using high-energy pulsed plasma accelerators [1], quasi-stationary plasma accelerators (QSPA) [2-7], and high-current electron beam accelerators $[8,9]$. These listed devices can simulate the desired loads on the materials. Using a pulsed plasma accelerator in model experiments makes it feasible to reproduce the magnitudes and time duration of heat loads characteristic of transient events in ITER. The impacts of the dust produced by the erosion of the thermonuclear fusion facility's first wall on the plasma are accompanied by impurities injected into the plasma. Therefore, in principle, the influence of dust on the collective behaviour of the plasma is of great interest, which requires investigating the mechanisms of dust formation, dust density, etc. [10]. In ITER for ELM events and reaction disruptions, high loads on the first wall are expected, which are difficult to be achieved in existing tokamaks. In this relation, pulsed plasma accelerators are more convenient setups to imitate these events in ITER on specific key parameters, test materials and investigate the characteristics of erosion products [11]. Pulsed plasma accelerators have also been widely used for the plasma processing of material surfaces. They enable the synthesis of various layers of impurity materials with appropriate adhesion to the treated surface on an unheated, cold substrate [12]. To this purpose, in 2013, we assembled and operated a single pulsed coaxial plasma accelerator at the Research Institute of Experimental and Theoretical Physics (IETP). For the improvement of operating parameters and energy efficiency of this setup, the constructional characteristics of electrodes were changed and the capacitance of energy storage capacitors was increased from $100 \mu \mathrm{F}$ up to 1.4 $\mathrm{mF}$. It was possible to obtain the required thermal flux, typical in Tokamaks. And also, these reconstructions and improvements in the experimental setup make it possible to obtain longer plasma pulses, which allows us to simulate the plasma filament and study its properties experimentally. For instance, the plasma disruption or ELMs (Edge Localized Modes) effects are the main problems in learning fusion facilities. Therefore, the main concern in the presented work is the experimental investigation of the pulsed plasma by various diagnostic methods after reconstructing the plasma accelerator, in particular, to estimate such essential parameters as, density and temperature of the electrons.

\section{Overview of the experimental setup}

The experiments were conducted on a coaxial pulsed plasma accelerator. A schematic diagram of an experimental setup is shown in Figure 1.

The central electrode (cylindrical anode) and the outer electrode (cylindrical cathode) of a pulsed plasma accelerator were made of copper $33 \mathrm{~cm}$ and $35 \mathrm{~cm}$ long and $5.5 \mathrm{~cm}$ and $10.8 \mathrm{~cm}$ in diameter, respectively. Also, the electrodes were electrically insulated from one another with a dielectric material. The overall electrode system was installed in a stainless steel vacuum chamber, and the fore vacuum and diffusion pumps were used to evacuate the chamber. The residual pressure limit before the experiment was about $5 \cdot 10^{-5}$ Torr. A capacitor battery 


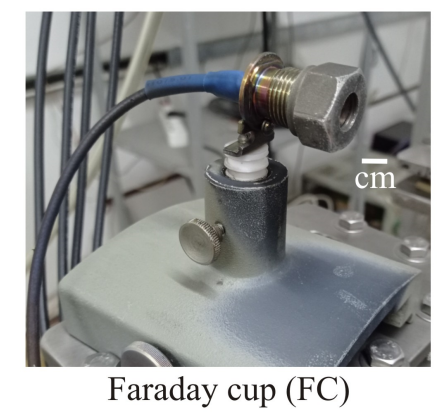
to the spectrometer

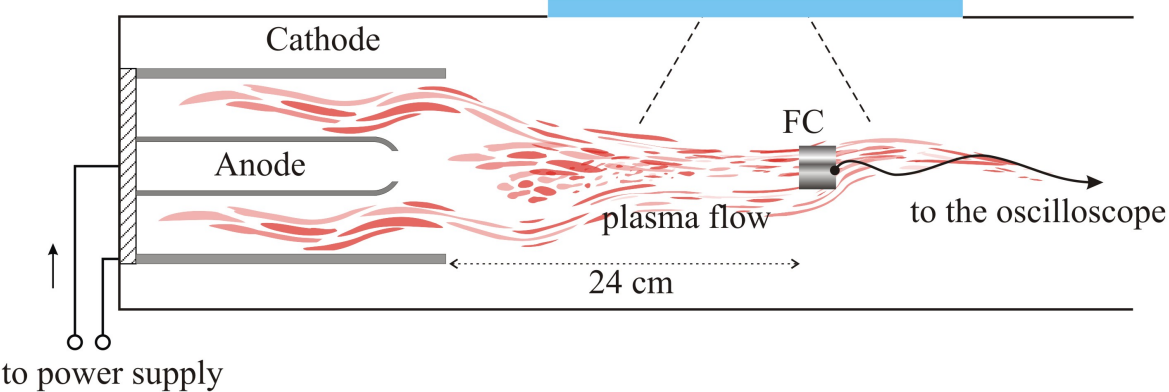

Figure 1. Schematic diagram of a coaxial pulsed plasma accelerator and measurement devices (top view).

with a capacitance of $1.44 \mathrm{mF}$, charged to $5 \mathrm{kV}$ and providing peak discharge currents of about 60-80 kA, was used to supply the setup. To evaluate the density and temperature of the electrons and ions in the plasma flow, during the breakdown and discharge phases, a Faraday cup (FC) was inserted inside the chamber on the path of the plasma flow, and plasma spectra were acquired from the side viewing windows on the exterior of the chamber. The Solar S-100 spectrometer was used to obtain plasma emission spectra. The optical range is from $190 \mathrm{~nm}$ to $1100 \mathrm{~nm}$, with an average spectral resolution of $1 \mathrm{~nm}$. The width of the spectrometer entrance slit is $20 \mu \mathrm{m}$. The signals from the Faraday cup were captured with a LeCroy WaveJet 354A four-channel digital oscilloscope, $\leq 500 \mathrm{MHz}$. In addition, a Phantom VEO710S high-speed camera was used in the experiment to observe the formation of the plasma flow. The camera was mounted perpendicular to the acceleration axis of the plasma flow (the camera viewing angle corresponds to the viewing angle of the spectrometer lens, as shown from Figure 1). So the accelerating plasma flow was observed from the side. The oscilloscope and high-speed camera sweep voltages from an external trigger with the signal under study were synchronized using a Berkeley Nucleonics Model 577 pulse generator.

The principle of operation of an experimental setup is as follows: once a controlled vacuum spark gap is actuated in the pulsed plasma accelerator's (PPA) electrical circuit, the energy stored in the capacitor battery is released through the electrodes in a time span that is usually a few microseconds. The voltage applied between the electrodes produces a strong electric field, causing the ionization of the gas and igniting an electric discharge, which rapidly expands into a plasma sheath. Then the plasma sheath is accelerated axially along the electrode node under the action of the Lorentz force $(J \times B)$, where $J$ is the radial component of the plasma sheath current density, and B is the azimuthally magnetic field of the plasma. Such a way the directed plasma flow is created. The average plasma 
pulse duration is $200 \mu \mathrm{s}$.

\section{Brief description of methods of plasma diagnostics}

A spectroscopic method of measuring the parameters of dynamic and spatially inhomogeneous pulsed discharges plasma is already well documented [13]. This method allows determining the plasma composition (including impurities), temperature and density of the electrons. The physical principles of this diagnostic method are as follows: plasma emits electromagnetic radiation as a result of electron transition between excited and ground states in the field of an atom or ion. Identification of plasma parameters is performed by measuring emission spectra intensities and analyzing the shape of spectral lines of this radiation. The main advantage of the method is the absence of perturbations of the investigated plasma and non-contact measurements. Like other indirect plasma diagnostics measurement methods, spectral measurement methods are also based on specific interpretation models. Since the plasma emission spectrum directly depends on the atomic processes in it, it is necessary to choose the model that will more accurately describe the real nature of the plasma. There are the following best-known plasma models: complete thermodynamic equilibrium, local thermodynamic equilibrium, corona balance model, collisional-radiative model, etc. In this paper, we will focus only on measurements in establishing local thermodynamic equilibrium (LTE). In the case of the LTE model, it is assumed that collision processes dominate in plasma, radiative losses are not too great, and electrons in energy levels in the atom are distributed according to Boltzmann's law. Therefore, the intensity of radiation during the transition of electrons from an excited state to an intermediate level or ground state with energy $E_{i}$ is defined as (1):

$$
I=N_{e} A_{e i} h v_{e i}=N_{0} \frac{g_{e}}{g_{0}} \exp \left(-\frac{E_{e}}{k T_{e}}\right) h v_{e i} A_{e i}
$$

where $h$ is Planck's constant, $v_{e i}$ - frequency of radiation, $A_{e i}$ - the transition probability. From expression (1), an intensity measurement allows us to estimate the temperatures of the electrons. The simplest method of calculating the temperature of the electrons is to measure the relative intensities of the two spectral lines $I_{1}$ and $I_{2}$ corresponding to two atomic (or two ionic) lines with wavelengths $\lambda_{1}$, $\lambda_{2}(2)$ :

$$
T_{e}=\frac{5040\left(\varepsilon_{1}-\varepsilon_{2}\right)}{\lg \left(\frac{g A_{1}}{g A_{2}}\right)-\lg \left(\frac{\lambda_{1}}{\lambda_{2}}\right)-\lg \left(\frac{J_{1}}{J_{2}}\right)},
$$

where $(g A)_{1}$ and $(g A)_{2}$ - emission transition probabilities, $\varepsilon_{1}$ and $\varepsilon_{2}$ are excitation energies. The essential criteria for the choice of spectral lines for this method is wavelength difference of 30-40 nm, which means that spectral lines should not belong to the same multiplet. Also the spontaneous transitions must be known.

To qualitatively estimate the density of the electrons in plasma, a spectral diagnostic method on the basis of Stark broadening of spectral lines is known. 
The physical basics of the method are as follows: in plasma, an emitting atom collides with the electrons, and the spectral line broadening occurs as a result of this collision [14]. For Stark broadened emission lines of hydrogen, the electron density can be calculated using the expression (3) and Griem's theory through the measurement of the spectral line width at the FWHM (full width half maximum) $\triangle \lambda_{1 / 2}$ :

$$
\Delta \lambda_{\frac{1}{2}}=2.50 \cdot 10^{-9} \alpha_{\frac{1}{2}} n_{e}^{\frac{2}{3}}
$$

where $\alpha_{1 / 2}$ is the theoretical value of the spectral line FWHM.

The apparatus for spectral measurement consists of three units: radiation source, optical lens (optical fiber), spectrometer and PC for spectra processing. Radiation is directed by the optical fiber to the spectrometer, which in turn separates it into monochromatic components according to wavelengths. The disadvantage of the spectral method of plasma diagnostics is that measurements are performed over a large volume of plasma.

To measure the distribution of charged particle fluxes in plasma, measurement methods based on different detectors are used. One of such detectors is Faraday cup $[15,16]$. Their principal advantage is their simplicity and portability. In some cases magnetic traps are used to prevent the influence of secondary electrons and reflection of dropping particles. A Faraday cup is a measuring device consisting of a cylindrical or conical geometry corpus on the outside of which an entrance aperture is made and inside of which there is a collector. The corpus and the collector are separated from each other by an insulator. The collector material is chosen so as to minimize the production of secondary electrons on its absorbing surface. The outer corpus is grounded; the collector is positively or negatively biased, according to what kind of charged particles we are dealing with. To perform measurements, the Faraday cup is placed in the direction of acceleration of the plasma flow. The density and temperature of the electrons and ions can be estimated from the measured current collected by the collector surface, depending on the bias voltage. The current-voltage characteristic (I-V curve) of the Faraday cup is also similar to that of a single Langmuir probe, so we will use the probe theory and its assumptions to evaluate the electron density and temperature: the particle energy distribution must be Maxwellian, the mean free path of the particles should be larger than the size of the collecting surface of the cup.

For determination of the density of particles expression (4) is used:

$$
n=\frac{J}{q v}
$$

where $J$ is current density, $q$ - particle charge, $v$ - particle velocity. The current density can be found as the ratio of the charged particle current to the aperture area $S$ on the collector. The ion velocity can be calculated by the Bohm criterion as (5):

$$
v_{i}=\sqrt{\frac{k T_{e}}{M_{i}}}
$$


where $T_{e}$ is temperature of the electrons obtained from I-V curve, $M_{i}$ is the mass of the ions.

\section{Results and Discussion}

Figure 2 shows instantaneous frames of the formation of the plasma filament and its time evolution, captured with a high-speed camera Phantom VEO710S. The acquisition rate is 280.000 frames / sec, at $256 \times 64$ camera resolution, with an exposure time of $3.1 \mu \mathrm{s}$. All frames shown in the figure were obtained in a single pulse, but at distinct points in time. The plasma acceleration in the figure is directed from the right to the left. We define the time of ignition of the arc discharge in the gap between the two electrodes as the initial point of reference. After ignition of the discharge, the discharge current creates a strong self-magnetic field. As a result, electromagnetic forces will act on the plasma and the plasma accelerates, then reaches the cathode tip and exits beyond it. It enters the visible area of the high-speed camera lens after $17.8 \mu$ s. Here, it should be noted that in one plasma pulse the filament is disrupted into several subfilaments which follow each other. The duration of the first subfilament and the directional velocity calculated from the two subsequent frames are $\sim 70 \mu$ s and $\vartheta \sim 25.5$ $\mathrm{km} / \mathrm{sec}$ respectively,. This is an evidence of the non-uniform plasma flow. The second subfilament appears after $96.4 \mu$ s, which is shown in Figure 2.

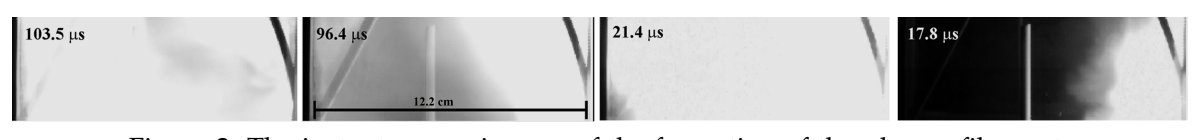

Figure 2. The instantaneous images of the formation of the plasma filament.

The emission spectra of the pulsed plasma flow were acquired using the S-100 linear spectrometer. The spectrometer operated in a single mode, with an exposure time of $50 \mathrm{~ms}$. Plasma radiation from the side viewing window was focused onto the entrance slit of the spectrometer via an optical condenser. The results of the optical-spectroscopic analysis of pulsed hydrogen plasma are shown in Figure 3.

Analysis of the lines shows the presence of the spectra of hydrogen of the Balmer series $\left(H_{\alpha}, H_{\beta}\right.$ and $\left.H_{\gamma}\right)$ and the lines of residual air (oxygen and nitrogen). Besides the primary spectra, spectral lines of iron are also present. Inside the chamber, the only region where iron is contained is the chamber vessel, which, in turn, is made of stainless steel. This indicates that the plasma energy, even at low voltages, is sufficient to vaporize the surface layer of the chamber material and excite the iron atoms. Moreover, a copper (CuII) line was observed, which appeared during plasma erosion of copper electrodes [17]. The intensity of spectral lines depends on the number of excitation and recombination acts. The latter increases with an increase in the applied energy and, according to the Paschen law, by matching the effective pressure value. For comparison, Figure 4 shows the emission spectrum of the pulsed air plasma flow. From Figure 4, it can be seen that when the voltage is increased to $5 \mathrm{kV}$, the intensity and number of spectral lines of the plasma grow. This is explained by the circumstance that with 


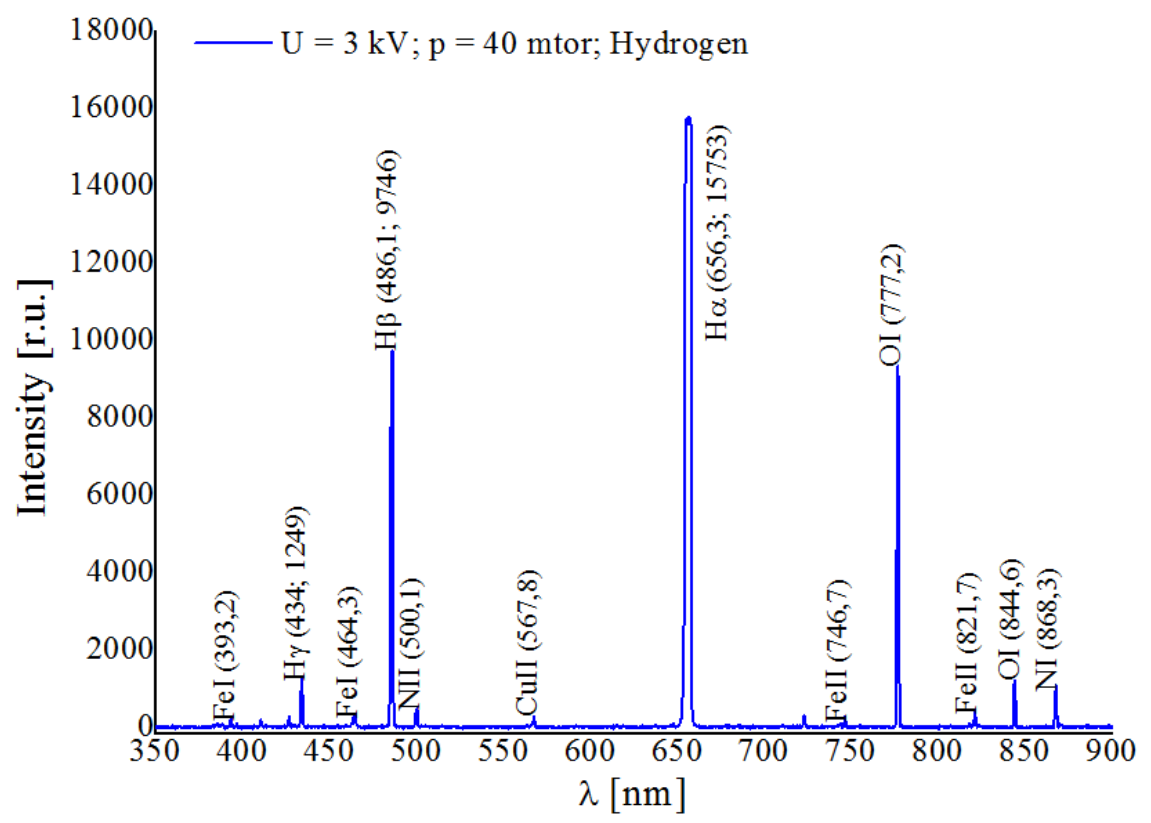

Figure 3. The emission spectra of the pulsed hydrogen plasma flow.

increasing voltage the energy of plasma particles moving along the accelerator expands, which increases the probability of excitation of atoms, thereby growing the intensity of glow spectral lines. Hydrogen spectra are also observed in the residual air, which may be related to the humidness of the air or to the erosion of the insulator, in our case caprolane, which separates the electrodes during the discharge.

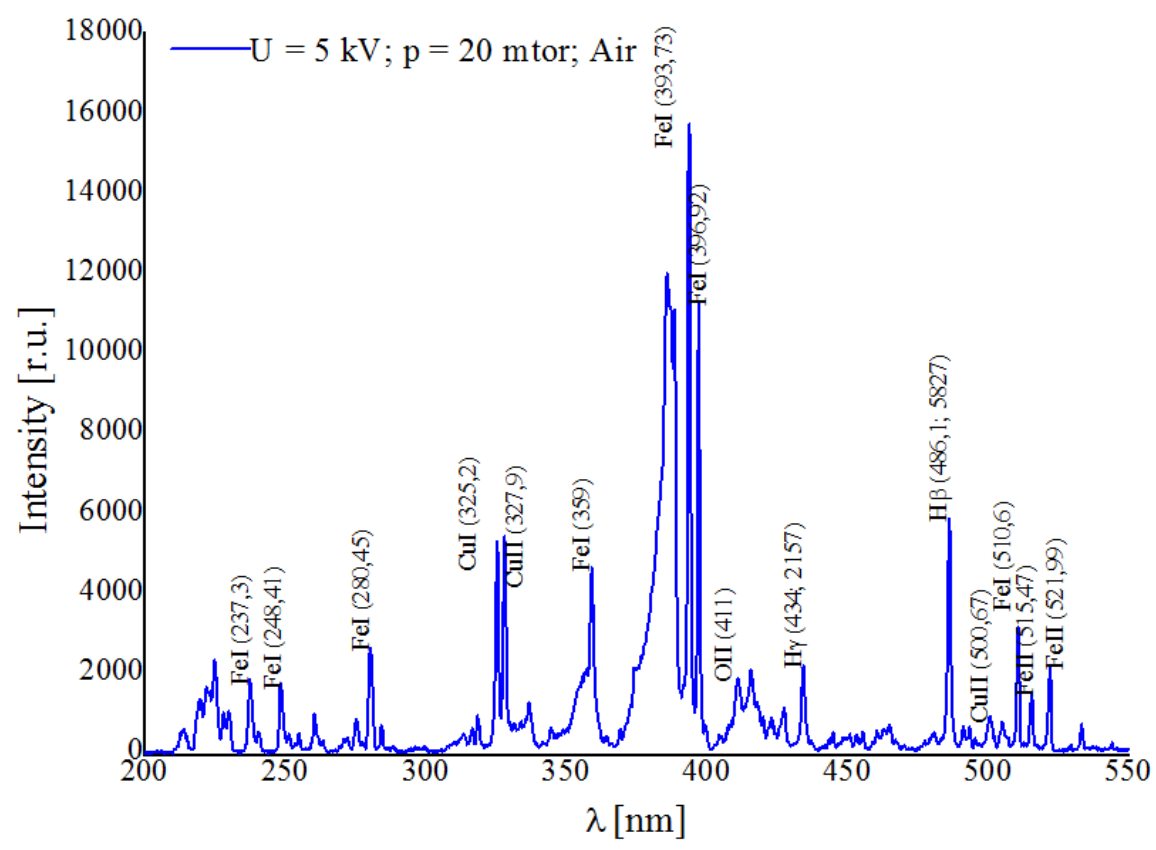

Figure 4 . The emission spectra of the pulsed air plasma flow.

The method for computing the temperature and density of the electrons in plasma is based on measuring the relative intensity of two spectral lines with different excitation energies of the atoms, and the difference in the excitation energy should not be lower than $2 \mathrm{eV}$. In this work we used the hydrogen spectral 
lines, more particularly the $H_{\alpha}$ and $H_{\beta}$ lines. So calculated by the expression (2) was $2.6 \mathrm{eV}$ for hydrogen plasma at a charging voltage of the capacitor bank of 3 $\mathrm{kV}$ and gas pressures of 40 mTor. If the temperature of the electrons is known, the density of the electrons can be determined as below using the expression (6):

$$
\lg n_{e}=-\lg \left(\frac{I_{i}}{I_{a}}\right)+\lg \left(\frac{(g A v)_{i}}{(g A v)_{a}}\right)-\frac{5040}{T}\left(V_{i}+\varepsilon_{i}-\varepsilon_{a}\right)+\frac{3}{2} \lg T+15.684,
$$

Also for the measurement of the electron density, the Stark broadening method of $H_{\beta}$ was used. For this reason the following experimental data obtained with the linear spectrometer S-100 and shown in Figure 5 and Figure 6 were used.

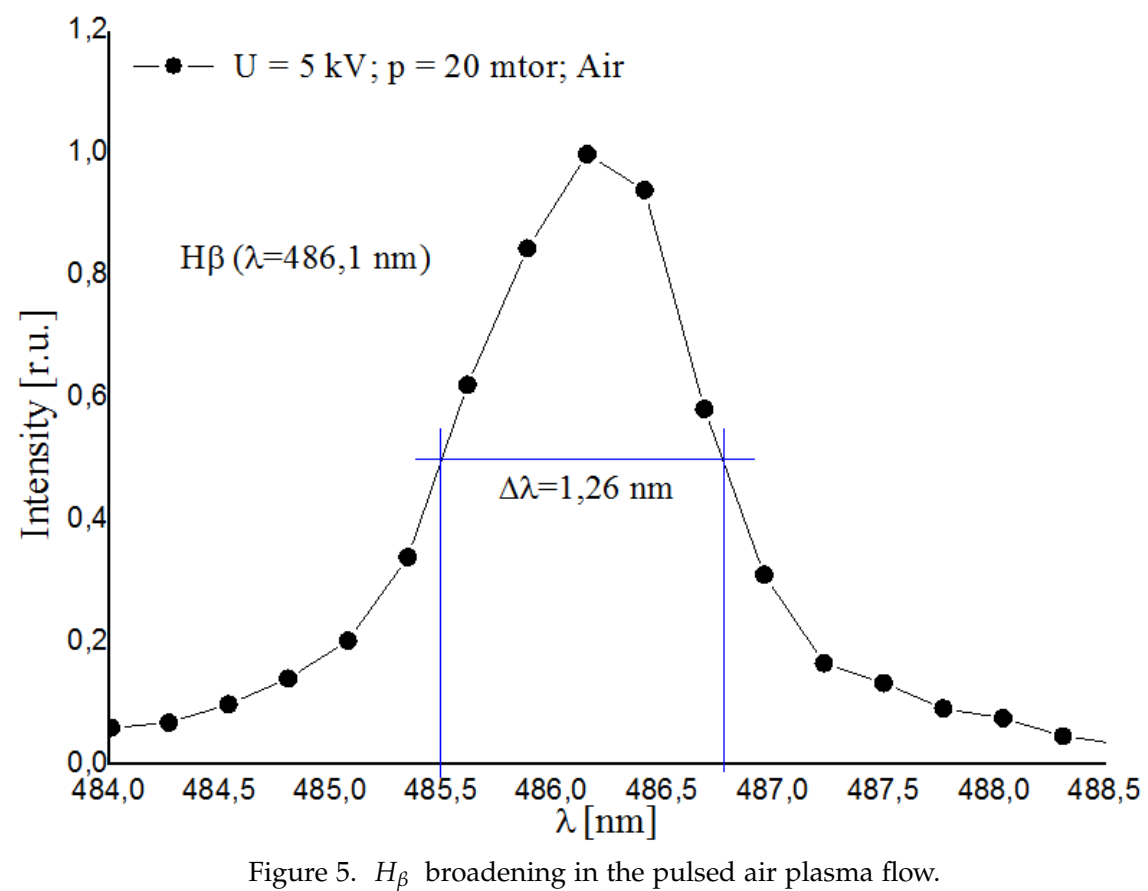

To achieve a more accurate result when measuring the width of the Stark broadening $H_{\beta}$ line with the Solar S-100 spectrometer, we took into account the instrumental width of the spectral apparatus [18], which forms the error of the optical system:

$$
\Delta \lambda_{\text {instr. }}=\sqrt{\Delta \lambda_{\text {slit }}^{2}+\Delta \lambda_{\text {resolution }}^{2}}=0.7 \mathrm{~nm}
$$

where $\Delta \lambda_{\text {slit }}$ is related to the dispersion of the diffraction grating and the width of the entrance slit of the spectrometer. So the calculated by us relatively valid half-widths of the Stark broadening $H_{\beta}$ line for air plasma is $\Delta \lambda_{1 / 2}=1.26-$ $0.7=0.56 \mathrm{~nm}$, and for hydrogen plasma is $\Delta \lambda_{1 / 2}=1.38-0.7=0.68 \mathrm{~nm}$. In that case the electron density in the pulsed air and hydrogen plasma flow calculated using the equation (3) were $4.3 \cdot 10^{15} \mathrm{~cm}^{-3}$ and $5.8 \cdot 10^{15} \mathrm{~cm}^{-3}$. Since the focus of this work is to practicing the methods of measuring plasma parameters [19], we will use the M833 monochromator with a spectral resolution of $0.022 \mathrm{~nm}$ for more accurate measurements in the future.

To compare these results in this work we also carried out electrical measurements based on a Faraday Cup [20, 21]. The Faraday Cup used in this work 


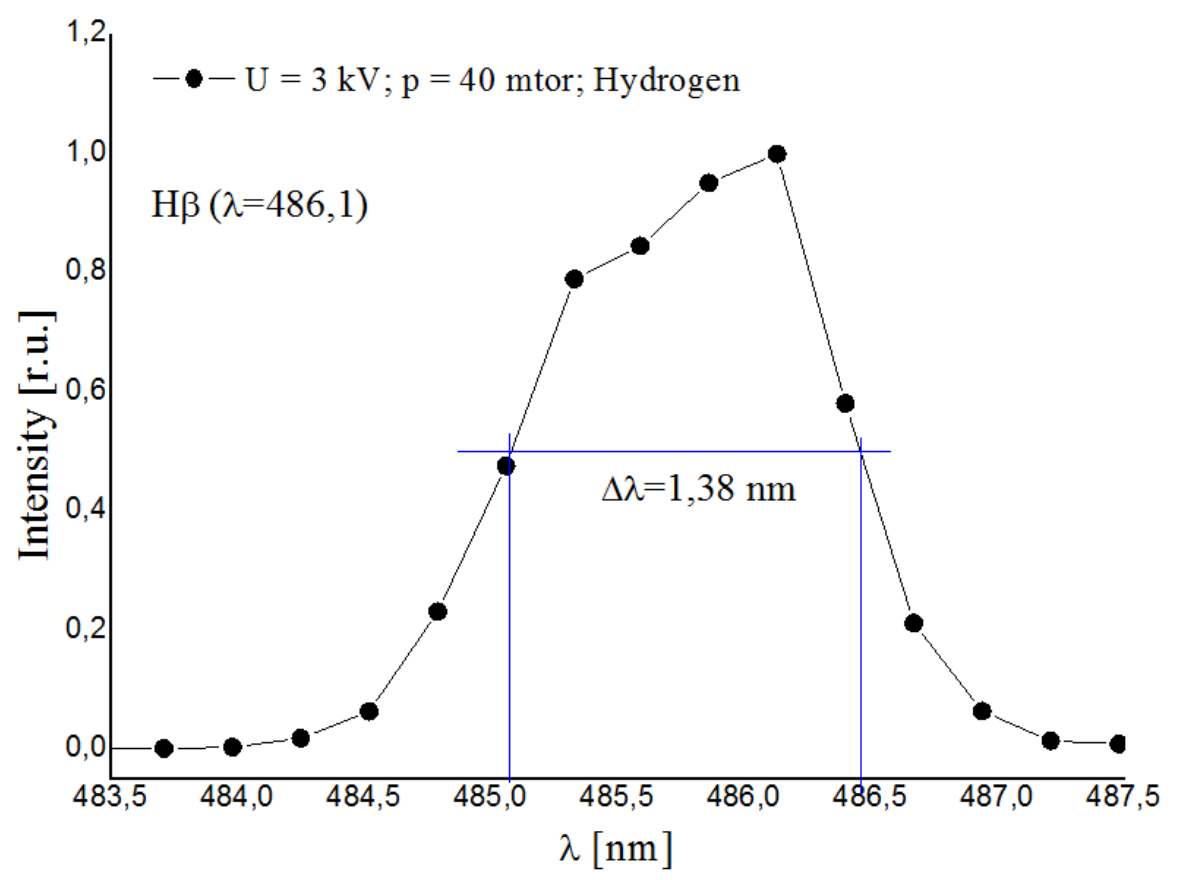

Figure 6. $H_{\beta}$ broadening in the pulsed hydrogen plasma flow.

consists of two coaxial cylindrical electrodes. Its collector is made from graphite, on the outer electrode an aperture with a diameter of $160 \mu \mathrm{m}$ is made to collect the charged particles. Graphite was chosen as the inner electrode because of its minimal secondary electron emission. A fluoroplastic insulator is used as the insulating material between the inner and outer electrodes. Furthermore, an internal electrode is supplied with an offset voltage of $+10 \mathrm{~V}$ to $+100 \mathrm{~V}$ and $-10 \mathrm{~V}$ to $-100 \mathrm{~V}$, respectively, and the outer electrode is grounded. FC was installed on the acceleration axis of the plasma flow at a $24 \mathrm{~cm}$ distance from the electrode system end. There is one of the side viewing windows at the same distance. Through it the optical emission spectrum of the plasma flow was captured by the spectrometer. During the spectral diagnostics, FC was removed from the investigation area. Figure 7 and 8 show the oscillograms of the ion and electron currents and the I-V characteristic of the electron current.

Figure 7 shows that the electrons attain the Faraday Cup before the ions, which indicates the division of charges in the plasma flow. The division of charges is due to the low mobility of ions.

To estimate the temperature of the electrons, the logarithmic scale of the I-V characteristic and expression (8) were used.

$$
T_{e}=\frac{e d U_{F C}}{k d \ln I_{e}}
$$

where $e$ - electrical charge, $k$ - Boltzmann constant, $U_{F C}$ - collector bias voltage. The calculated electron temperature, using the slope of the I-V curve, at a capacitor bank charging voltage of $3 \mathrm{kV}$ and gas pressures of 40 mTor for the pulsed hydrogen plasma flow was $23.6 \mathrm{eV}$. According to the expressions 5 and 4 , by the temperature of the electrons we have found the density of electrons and ions.

The obtained results of electron density and temperature by two methods: spectral plasma diagnostics and electrical measurement based on a Faraday Cup 


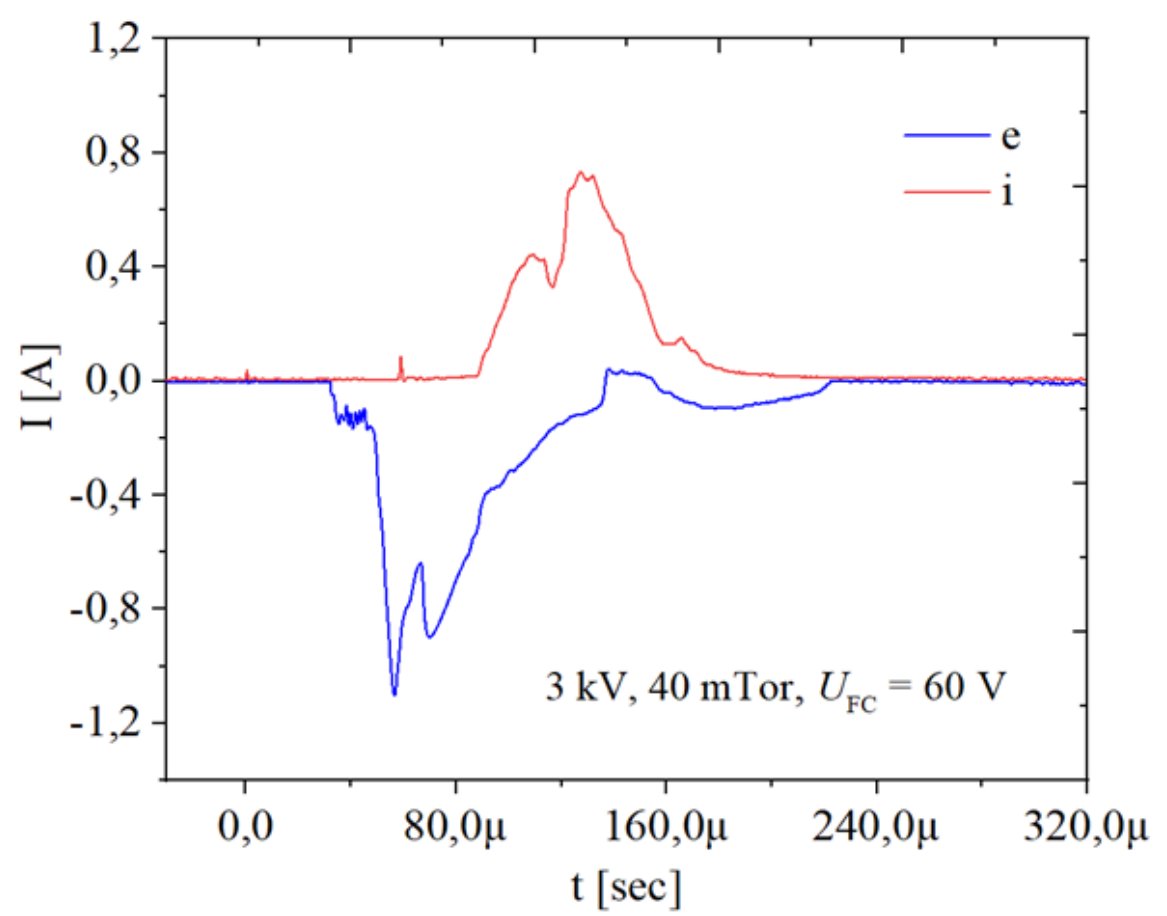

Figure 7. Oscillograms of ion and electron currents in the pulsed hydrogen plasma flow.

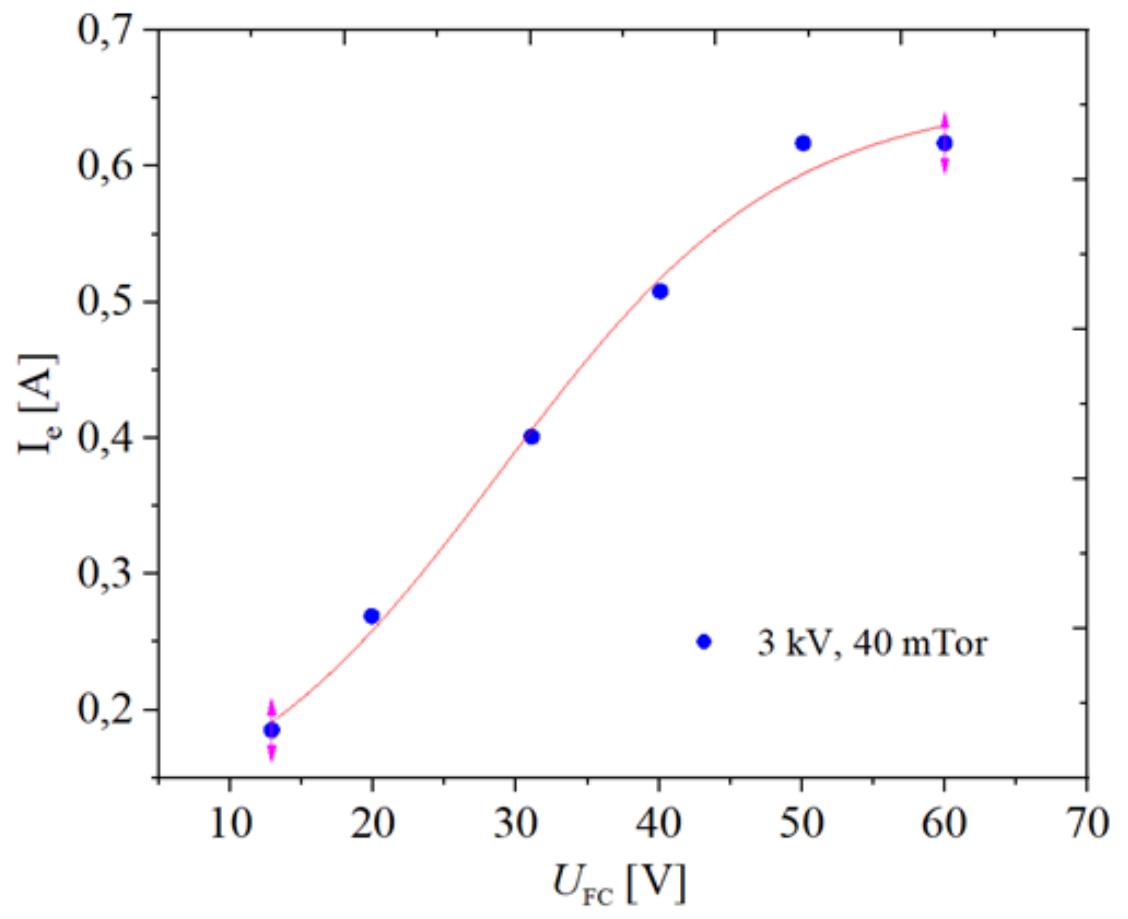

Figure 8. I-V characteristic of the pulsed hydrogen plasma flow.

for comparison are presented in Table 1. 
Table 1.

Results of measurements of electron density and temperature in the pulsed hydrogen plasma flow.

\begin{tabular}{l|l|l|l|}
\hline & $T_{e}[\mathrm{eV}]$ & $n_{e}\left[\mathrm{~cm}^{-3}\right]$ & $n_{i}\left[\mathrm{~cm}^{-3}\right]$ \\
\hline Spectral diagnostics & 2.6 & $5.8 \cdot 10^{15}$ & \\
\hline Faraday Cup & 23.6 & $7.4 \cdot 10^{13}$ & $3.2 \cdot 10^{15}$ \\
\hline
\end{tabular}

This work is a continuation of [22], where using a triple electric probe, in real time and in a single plasma pulse it was successfully measured the temperature and density of the electrons. The principles of measurement with Faraday cup are based on the theory of a single electric probe, so the problem of plotting the real I-V curve appears. Since each point of the I-V curve corresponds to a separate pulse, where their parameters may differ from each other, which leads to significant errors. In the future it is planned to develop this method and perhaps find another way to calculations that differs from the single probe theory and allows measuring the plasma flow parameters in a single pulse. In besides, the method is contact, therefore, electric and magnetic fields, created by plasma, may introduce errors in measurements of 30-40 \% [23]. Compared with the spectral method, the Faraday cup measures the particle flux density on the surface, for instance, where the target material under study would be located. In this regard, the results of the work are particularly important when studying the processes of melting, erosion, sputtering, and degradation of the surfaces of candidate materials of the first wall of fusion reactors during their interaction with high-energy plasma.

The advantages of optical emission spectroscopy are the non-contact measurement and the absence of perturbation in the plasma. As mentioned above, we tried to subtract the instrumental error; in addition, according to the known sources on spectroscopy in a careful study, the error of this method does not exceed $10 \%[24,25]$.

\section{Conclusion}

In a coaxial plasma accelerator, pulsed plasma diagnostics by optical emission spectroscopy and Faraday cup were performed to measure plasma parameters and experimentally model processes in thermonuclear fusion reactors. To obtain the emission spectra of plasma radiation in the wavelength range of 350-900 $\mathrm{nm}$ a linear diffraction spectrometer was used. The following intense lines were observed: hydrogen Balmer series $\left(H_{\alpha}, H_{\beta}, H_{\gamma}\right)$, copper (CuII-567.8 nm), iron (FeII-821.7 nm, FeI-393.2 nm) and residual air (NI-868.3 nm, OI-844.6 nm). Using the Balmer spectral lines, the temperature and density of charged particles in the plasma flow were obtained (for duration of the existence of the plasma flow).

Using a Faraday cup (FC), the I-V characteristic of the pulsed plasma was obtained to calculate the electron temperature, electron and ion densities in the localized plasma region. Compared with the spectral method, the FC is in contact with the plasma and measures the particle flux density on its surface. 
The results are significant for studying the processes of melting, erosion, sputtering, and degradation of the surfaces of candidate materials of the first wall of thermonuclear fusion reactors during their interaction with high-energy plasma flux.

\section{Acknowledgments}

This work was supported by the Ministry of Education and Science of the Republic of Kazakhstan (project no. IRN AP08053373).

\section{References}

[1] I.M. Poznyak et al., AIP Conference Proceedings 1771 (2016) 060006.

[CrossRef]

[2] N.S. Klimova et al., Journal of Nuclear Materials 463 (2015) 61-65. [CrossRef]

[3] I. Garkusha et al., IOP Conf. Series: Journal of Physics: Conf. Series 959 (2018) 012004. [CrossRef]

[4] A. Suslova et al., Nuclear Fusion 55 (2015) 033007. [CrossRef]

[5] V.A. Makhlaj et al., Journal of Nuclear Materials 438 (2013) S233-S236.

[CrossRef]

[6] I.E. Garkusha et al., Journal of Nuclear Materials 386-388 (2009) 127-131. [CrossRef]

[7] I.E. Garkusha et al., Journal of Nuclear Materials 337-339 (2005) 707-711. [CrossRef]

[8] J. Linkea et al., Matter and Radiation at Extremes 4 (2019) 056201. [CrossRef]

[9] N. Sorokina et al., Nuclear Instruments and Methods in Physics Research

Section A: Accelerators, Spectrometers, Detectors and Associated Equipment 623 (2010) 750-753. [CrossRef]

[10] S. Krasheninnikov et al., Plasma Phys. Control. Fusion 57 (2015) 044009. [CrossRef]

[11] M. Dosbolayev et al., IEEE Transaction on plasma science 7 (2019) 3047-3051. [CrossRef]

[12] K. Nowakowska-Langier et al., Appl. Surf. Sci. 275 (2013) 14-18. [CrossRef]

[13] R. Hadlstone, Plasma diagnostics (Mir, Moscow, 1967) 516 p. (In Russian)

[14] V. Locht-Holtgreven, Methods of plasma examination (Mir, Moscow, 1971)

126 p. (In Russian)

[15] S.Yu. Lukyanov, Hot Plasma and Controlled Nuclear Fusion (Nauka, Moscow, 1975) 398 p. (In Russian)

[16] P. Youn Duck-Sang, Measurements on laser produced plasma using Faraday-cups (Monterrey, California: Springfield, 1989) 80 p. [Google Scholar]

[17] B. Kulakowska-Pawlak, Plasma Sources Sci. Technol. 18 (2009) 035015. [CrossRef]

[18] T. Vaczi, Applied Spectroscopy 68 (2014) 1274-1278.[CrossRef]

[19] A. Tazhen, et al., Vestnik KAZNRTU 3 (2020) 153-158. (In Russian)

[20] Lian-Kuang Lim et al., Plos one 13 (2018) e0188009. [CrossRef] 
210 Eurasian Journal of Physics and Functional Materials, Vol.5(4).

[21] M. Habibi, Physics Letters A 380 (2016) 439-443. [CrossRef]

[22] A. Tazhen et al., Plasma physics reports 46 (2020) 153-158. [CrossRef]

[23] F.F. Chen, Physics of Plasmas 8 (2001) 3029. [CrossRef]

[24] V.N. Ochkin, Spectroscopy of Low-Temperature Plasma (Wiley, Germany, 2009 ) 630 p. [Google books]

[25] J. Wiechula. et al., Physics of Plasmas 22 (2015) 043516. [CrossRef] 